\title{
Optimization of the feeding rate of Anopheles farauti s.s. colony mosquitoes in direct membrane feeding assays
}

\author{
Lincoln Timinao ${ }^{1,2^{*}}$ (D), Rebecca Vinit ${ }^{1}$, Michelle Katusele ${ }^{1}$, Louis Schofield ${ }^{2}$, Thomas R. Burkot ${ }^{2}$ and
} Stephan Karl ${ }^{1,2}$

\begin{abstract}
Background: Direct membrane feeding assays (DMFA) are an important tool to study parasite transmission to mosquitoes. Mosquito feeding rates in these artificial systems require optimization, as there are a number of factors that potentially influence the feeding rates and there are no standardized methods that apply to all anopheline species.

Methods: A range of parameters prior to and during direct membrane feeding (DMF) were evaluated for their impact on Anopheles farauti sensu stricto feeding rates, including the starving conditions and duration of starving prior to feeding, membrane type, DMF exposure time, mosquito age, feeding in the light versus the dark, blood volume, mosquito density and temperature of water bath.

Results: The average successful DMFA feeding rate for An. farauti s.S. colony mosquitoes increased from 50 to $85 \%$ when assay parameters were varied. Overnight starvation and Baudruche membrane yielded the highest feeding rates but rates were also affected by blood volume in the feeder and the mosquito density in the feeding cups. Availability of water during the pre-feed starvation period did not significantly impact feeding rates, nor did the exposure duration to blood in membrane feeders, the age of mosquitoes ( 3,5 and 7 days post-emergence), feeding in the light versus the dark, or the temperature $\left(34^{\circ} \mathrm{C}, 38^{\circ} \mathrm{C}, 42^{\circ} \mathrm{C}\right.$ and $\left.46^{\circ} \mathrm{C}\right)$ of the water bath.

Conclusion: Optimal feeding conditions in An. farauti s.s. DMFA were to offer 50 female mosquitoes in a cup (with a total surface area of $\sim 340 \mathrm{~cm}^{2}$ with 1 mosquito $/ 6.8 \mathrm{~cm}^{2}$ ) that were starved overnight 350-500 $\mu \mathrm{L}$ of blood (collected in heparin-coated Vacutainer tubes) per feeder in feeders with a surface area $\sim 5 \mathrm{~cm}^{2}$ (with a maximum capacity of $1.5 \mathrm{~mL}$ of blood) via a Baudruche membrane, for at least 10-20 min.
\end{abstract}

Keywords: Direct membrane feeding assay, Anopheles farauti, Papua New Guinea

\section{Background}

In the malaria parasite life cycle, transmission through the mosquito vector represents a bottleneck where parasite populations shrink from millions in the human body to as few as one in the mosquito [1]. Thus, malaria transmission is vulnerable to interruption when transiting

\footnotetext{
*Correspondence: lincoln.timinao@gmail.com

${ }^{1}$ Papua New Guinea Institute of Medical Research, Madang, Papua New Guinea
}

Full list of author information is available at the end of the article from the human to the mosquito host [2]. This transition can be studied by direct membrane feeding assays (DMFA). During DMFA, mosquitoes feed through a membrane on blood kept warm via water-jacketed glass feeders [3] including blood harvested from humans with circulating malaria parasites [4], to either study parasite development in the mosquito [4] or to test interventions that disrupt parasite development, hence interrupting transmission [5].

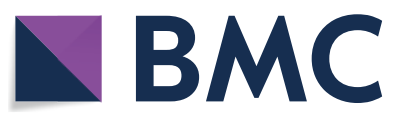

(c) The Author(s) 2021. This article is licensed under a Creative Commons Attribution 4.0 International License, which permits use, sharing, adaptation, distribution and reproduction in any medium or format, as long as you give appropriate credit to the original author(s) and the source, provide a link to the Creative Commons licence, and indicate if changes were made. The images or other third party material in this article are included in the article's Creative Commons licence, unless indicated otherwise in a credit line to the material. If material is not included in the article's Creative Commons licence and your intended use is not permitted by statutory regulation or exceeds the permitted use, you will need to obtain permission directly from the copyright holder. To view a copy of this licence, visit http://creativeco mmons.org/licenses/by/4.0/. The Creative Commons Public Domain Dedication waiver (http://creativecommons.org/publicdomain/ zero/1.0/) applies to the data made available in this article, unless otherwise stated in a credit line to the data. 
The mosquito blood feeding rate, i.e., the proportion of mosquitoes that successfully ingest blood, is an important determinant of overall infection success. The success rate of ingesting blood from a membrane feeder can vary depending on the mosquito species, whether the mosquitoes were collected in the wild [6] or reared in a colony [7], as well as the level of adaptation of the colony.

Blood feeding rates also depend on the experimental conditions under which the DMFAs are conducted, including (i) the duration of starvation before exposure, (ii) the starving conditions (access to water or no access to water), (iii) the type of membrane used, (iv) the amount of time mosquitoes are allowed to feed, (v) the mosquito age, (vi) feeding in the light or in the dark, (vii) the blood volume in the feeder, (viii) the density of mosquitoes attempting to feed and (ix) water bath temperature during DMFA (Fig. 1). Other parameters which also potentially influence blood-feeding rates in DMFA but were not investigated here include the blood meal source [8], the haematocrit level, [9] and phagostimulants such as sodium chloride and sodium bicarbonate for Anopheles species [10].

However, membrane feeding studies have been conducted with a range of conditions and with varied feeding success $[11,12]$. Thus, there is a need to optimize DMFA conditions for each colony mosquito species.

Starving conditions are a key component that greatly impact mosquito feeding rates and a balance needs to be established between starving the mosquitoes for too long, thereby increasing mosquito mortality or affecting their fitness [13], and not starving for long enough so mosquitoes only partly feed or not at all.

Most studies describe dry starving for durations from 5 to $36 \mathrm{~h}$ [12, 14-16] while other studies performed starving where the mosquitoes had access to water for

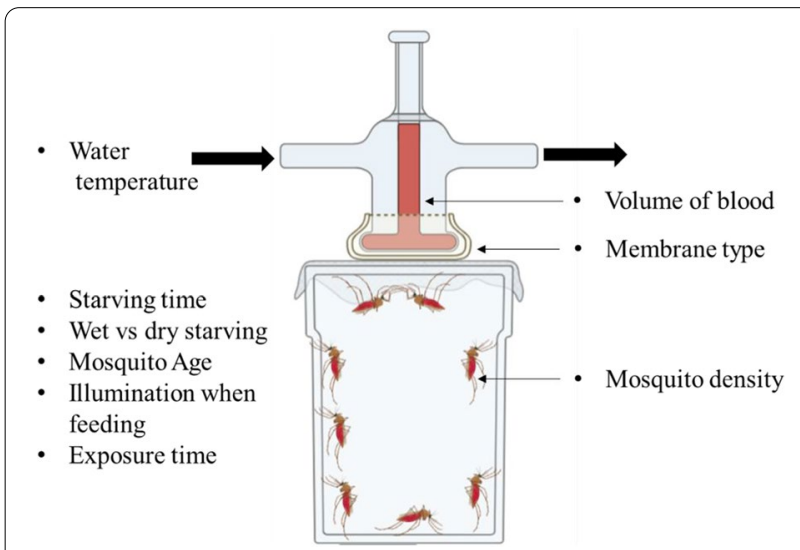

Fig. 1 Membrane feeding assay set-up with the parameters impacting the feeding success of mosquitoes on the direct membrane feeders (figure created using BioRender.com)
$12 \mathrm{~h}[17,18]$. A study conducted by Coulibaly and colleagues compared the feeding rates of mosquitoes dry starved $8 \mathrm{~h}, 14 \mathrm{~h}$ and $20 \mathrm{~h}$ and concluded that mosquitoes starved $8-14 \mathrm{~h}$ yielded significantly higher feeding rates than mosquitoes starved $20 \mathrm{~h}$ [13]. However, most studies did not directly report the impact of starving on the feeding rate.

Membranes take the role of an artificial skin in the feeding experiments. An ideal membrane will yield the highest feeding rates in the shortest period of time. Parafilm and natural membranes such as Baudruche, sausage casing, chicken skin, or rat skin have been used $[6,19]$. Natural membranes which closely mimic the skin resulted in the highest feeding rates followed by Baudruche membrane, which is derived from bovine cecum, and finally Parafilm, a wax synthetic membrane [3]. Most studies reported using Baudruche membrane $[11,20,21]$ while others used Parafilm membrane [16, $22]$. Interestingly, a study done by Coulibaly and colleagues showed that there was no significant difference between the feeding rates, survival and infection rates from feeding experiments with either Baudruche or Parafilm membranes, for Anopheles coluzzii mosquitoes [13].

Mosquitoes 2-8 days post-emergence have been used in different studies $[6,7,12,13,16,17,23-25]$. The main consideration in this is that mosquitoes are fed at an early age so that they survive for the required duration for either oocysts $[11,12,16,26,27]$ or sporozoites [11, $26,27]$ to develop. The study by Coulibaly and colleagues is so far the only one that compared the feeding rate of An. coluzzii mosquitoes between 3 and 9 days post emergence. The authors determined that 3-day-old mosquitoes had a significantly higher feeding rate compared to 6 and 9-day-old mosquitoes [13].

Mosquito density is another factor that may influence the mosquito feeding rate. Rutledge and colleagues observed that having more mosquitoes per cage can result in lower feeding rates [3] and crowding, making handling, especially removing of unfed mosquitoes, difficult. Vallejo and colleagues observed that $100 \mathrm{An}$. coluzzii mosquitoes per cage (or 1 mosquito per $5 \mathrm{~cm}^{2}$ ) resulted in the highest Plasmodium vivax infection prevalence after DMFA [27]. However, the study did not report on the feeding rate of the different mosquito densities in relation to infection success.

Little has also been reported with respect to the impact of the other parameters listed above on the feeding rates. Much of the focus is on the infection rates. Therefore, the focus of this study was to determine the optimal feeding conditions for Anopheles farauti sensu stricto colony mosquitoes in order to maximize their feeding rates in DMFA. 


\section{Methods}

\section{Mosquito colony maintenance}

The $A n$. farauti s.s. mosquito colony was derived from a colony established in 1968 in Rabaul, East New Britain province. [28] The laboratory colony was maintained at $28 \pm 8{ }^{\circ} \mathrm{C}$ and $68 \pm 25 \%$ relative humidity. The light cycle is approximately $11 \mathrm{~h}$ dark and $12 \mathrm{~h}$ light, including a 30 min dusk and 30 min dawn period. The larvae were fed ground fish food (Marine Master Tropical Fish Flakes, Australia) while the adults were provided 10\% sucrose (Ramu Sugar, Papua New Guinea) solution available as soaked cotton wool balls placed on top of the mosquito cages. To maintain the colony, uninfected blood (no malaria) was obtained from donors following informed consent procedure. Occasionally, direct skin feeding was used to maintain the colony.

\section{Direct membrane feeding assays}

Water-jacketed glass membrane feeders were connected in series by rubber hoses to a mini aquarium pump placed inside a $37-38{ }^{\circ} \mathrm{C}$ water bath (Fig. 2). Unless otherwise stated, all trials used an average of 5-day-old mosquitoes with 50 female $A n$. farauti s.s. placed in a cup (surface area of $\sim 340 \mathrm{~cm}^{2}$ with a total volume of $\sim 476$ $\mathrm{cm}^{3}$ ) and offered blood meals from feeders with a diameter of $2.5 \mathrm{~cm}$ (a surface area of $\sim 5 \mathrm{~cm}^{2}$ with a maximum blood volume capacity of $1 \mathrm{~mL}$ ). The duration of overnight starving ranged between 18 and $21 \mathrm{~h}$.

The experiments were done sequentially with a single parameter being varied and tested, incorporating the optimal conditions of the preceding tests. Following the feeding experiments, unfed mosquitoes were separated from the fully fed mosquitoes and the feeding rate calculated. Data were transformed using arcsine (p) [29] prior to performing paired $t$ tests to test the significance of the

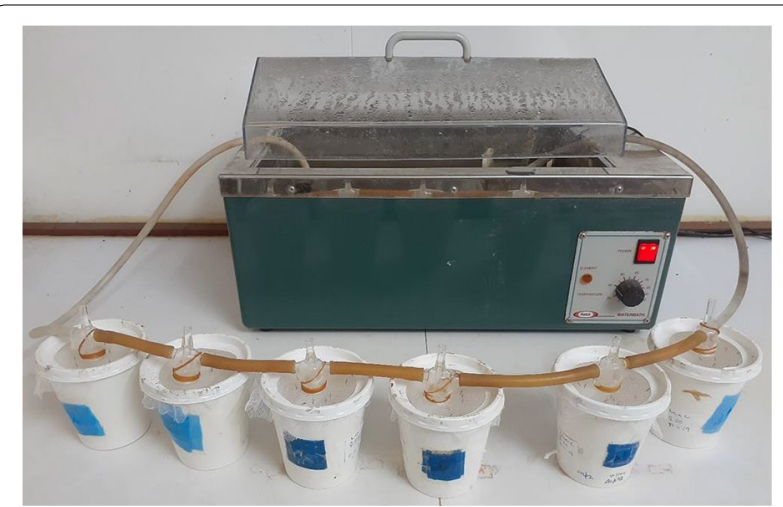

Fig. 2 Direct membrane feeding assay (DMFA) set-up. Cups connected in a series by tubes to a mini aquarium pump within the water bath which is maintained at $\sim 38^{\circ} \mathrm{C}$ difference observed between groups. Analysis of variance (ANOVA), followed by $t$ tests, was used to test for significant variation between more than two groups. A flow chart summarizing the parameters tested is provided in Additional file 1: Table S1.

\section{Starving time}

An. farauti s.s. were dry starved (no access to sugar or water) for $2 \mathrm{~h}, 4 \mathrm{~h}, 6 \mathrm{~h}$ and overnight $(\sim 21 \mathrm{~h})$ and compared to a control of mosquitoes exposed without starving. Mosquitoes were exposed to $\sim 750 \mu \mathrm{L}$ of blood for $30 \mathrm{~min}$ in the dark (with a black piece of blanket draped over the membrane feeder) following starvation. Parafilm membrane (cut into $3 \mathrm{~cm} \times 3 \mathrm{~cm}$ and stretched to $\sim 5 \mathrm{~cm} \times \sim 5 \mathrm{~cm}$ ) was used to feed the mosquitoes. The Parafilm was not exposed to human odour prior to feeding. Ten replicates were performed.

\section{Type of starving: access to water versus dry (no access to water)}

An. farauti s.s. mosquitoes were starved overnight, with one cup of mosquitoes having access to cotton soaked in water while the other did not have access to water (dry starved). The mosquitoes were then given access to $\sim 750$ $\mu \mathrm{L}$ of blood using a Parafilm membrane for $30 \mathrm{~min}$ in the dark. Five replicates were performed.

\section{Membrane type}

Two membrane types were tested, namely Parafilm and Baudruche membranes. The Parafilm membrane was standardized by cutting it into $3 \mathrm{~cm} \times 3 \mathrm{~cm}$ pieces and stretching to $\sim 5 \mathrm{~cm} \times \sim 5 \mathrm{~cm}$. Mosquitoes were dry starved overnight. The mosquitoes were given access to $\sim 750 \mu \mathrm{L}$ of blood in a membrane feeder for $30 \mathrm{~min}$ in the dark. Eight replicate experiments were performed.

\section{Exposure time}

Exposure times of $10 \mathrm{~min}, 20 \mathrm{~min}$ and $30 \mathrm{~min}$ were evaluated following dry overnight starving and the mosquitoes were then exposed to $\sim 750 \mu \mathrm{L}$ of blood for the specified time. Baudruche membrane was used to feed the mosquitoes. Eight replicate experiments were performed.

\section{Mosquito age}

Mosquitoes aged 3, 5 and 7 days were tested in nine replicates. The mosquitoes were starved overnight before exposure to $\sim 750 \mu \mathrm{L}$ of blood via a Baudruche membrane for $20 \mathrm{~min}$ in the dark.

\section{Feeding in the light or in the dark}

A total of 2 cups of mosquitoes were prepared and starved overnight. One cup of mosquitoes was fed with the net top exposed to ambient room lighting, while 
the second cup had a black blanket placed over it while they were exposed to $\sim 750 \mu \mathrm{L}$ of blood via Baudruche membrane for $20 \mathrm{~min}$. Seven replicate experiments were performed.

\section{Volume of blood}

Blood volumes of $125 \mu \mathrm{L}, 250 \mu \mathrm{L}$ and $500 \mu \mathrm{L}$ in a waterjacketed glass feeder of $1.5 \mathrm{~mL}$ maximum capacity were tested to determine the minimum blood volume which could yield high feeding rates. Three cups of 6-day-old (mean age) An. farauti s.s. were prepared, starved overnight and allowed $20 \mathrm{~min}$ to feed in the dark at each blood volume via a Baudruche membrane. Six replicate experiments were performed.

\section{Mosquito density}

Three different mosquito numbers, i.e., 20, 50 and 100 per cup, were tested. Mosquitoes at a mean age of 4 days were dry starved overnight. The mosquitoes were allowed to feed on $\sim 500 \mu \mathrm{L}$ of blood for 20 min under illuminated conditions via a Baudruche membrane. Six replicates were performed for this test.

\section{Water temperature}

Four different water bath temperatures were tested, i.e., $34{ }^{\circ} \mathrm{C}, 38^{\circ} \mathrm{C}, 42{ }^{\circ} \mathrm{C}$ and $46^{\circ} \mathrm{C}$. Mosquitoes were dry starved overnight before being allowed to feed on $\sim 500$ $\mu \mathrm{L}$ of blood for 20 min under illuminated conditions via a Baudruche membrane. Seven replicates were performed for this test.

\section{Results}

The volunteers who donated blood for membrane feeding were adults with a median age of 46 (range of 28-52) and a median haemoglobin level of $15.5 \mathrm{~g} / \mathrm{dL}$ with the range of $11.1-17.7 \mathrm{~g} / \mathrm{dL}$. The median room temperature was $28.05{ }^{\circ} \mathrm{C}$ (range of $22.2-30.34{ }^{\circ} \mathrm{C}$ ) with a relative humidity of $78.38 \%$ (range of $56-89.3 \%$ ). The baseline parameters to which subsequent tests were compared were: 50 female $A n$. farauti s.s. per cup, aged between 3 and 5 days, dry starved between 0 and $4 \mathrm{~h}$, and fed in the dark using Parafilm as the membrane for $\sim 30 \mathrm{~min}$, with $750 \mu \mathrm{L}$ of blood at a water bath temperature of $\sim 38^{\circ} \mathrm{C}$.

Table 1 summarizes the results of the analysis for the starving duration, type of starving, membrane type, feeding duration, mosquito age, feeding in the light versus in the dark, volume of blood, mosquito density and water bath temperature.

There was a statistically significant difference observed between the feeding rates of the different starving times as determined by one-way ANOVA $(F(3,24)=8.982$, $p<0.001)$. When performing $t$ tests for the different paired groups, we found that there were significant differences between the overnight starving and the other starving times (Fig. 3), with feeding rates approximately doubled when comparing from $0 \mathrm{~h}(27 \%)$ to $\sim 21 \mathrm{~h}(60 \%)$. The differences in the feeding rates after starving the mosquitoes for $6 \mathrm{~h}, 4 \mathrm{~h}$ and $2 \mathrm{~h}$ were not statistically significant $(p=0.51, p=0.10, p=0.18)$. We observed a mortality rate of $7 \%$ with the overnight starvation compared to the other groups, which exhibited an average mortality of $1 \%$. The observed difference between the mortality rates at starving times of $0 \mathrm{~h}, 2 \mathrm{~h}, 4 \mathrm{~h}, 6 \mathrm{~h}$ and overnight is statistically significant as determined by the one-way ANOVA $(F(3,19)=4.000, p=0.03)$. There was no significant difference observed between the type of starving, i.e., whether the mosquitoes were dry starved or allowed to feed on water during the starving period ( $p=0.47)$.

There was a statistically significant difference in the feeding rates for the two types of membranes tested, Baudruche and Parafilm $(p<0.05)$ (Fig. 4). Feeding rate increased to $85 \%$ when the Baudruche membrane was used.

Exposure times, mosquito age or feeding in the light versus in the dark were not observed to significantly influence membrane feeding rates (Table 1). However, there was a statistically significant difference in the feeding rates for the different volumes of blood as determined by one-way ANOVA $(F(2,10)=13.70, p<0.01)$. We observed an increase in the feeding rate when the blood volume was increased from $125 \mu \mathrm{L}$ to anything above $250 \mu \mathrm{L}$. The difference was statistically significant $(p<0.01)$ (Fig. 5). The differences in the feeding rates at different mosquito densities approached significance as determined by one-way ANOVA $(F(2,15)=3.861$, $p=0.052$ ). When comparing different mosquito density groups, we observed that feeding rates were higher for cups with a mosquito density of 50 per cup as compared to 100 per cup $(p<0.01)$ (Fig. 6). However, there was no significant difference between 50 and 20 mosquitoes per cup $(p=0.13)$, indicating that 50 mosquitoes per cup was closer to the optimal mosquito density. Interestingly, we did not observe any statistically significant difference in the feeding rate between 20 and 100 mosquitoes.

There was no statistically significant difference between the feeding rates at different exposure times (10, 20 and $30 \mathrm{~min}$ ), the different mosquito ages (3, 5 and 7 days old) and the different water bath temperatures (34, 38, 42 and $\left.46^{\circ} \mathrm{C}\right)$ as determined by one-way ANOVA ( $F(1$, $9)=0.3522, \quad p=0.63, \quad F(2,13)=1.197, p=0.32, \quad F(1$, $9)=0.3522, p=0.63)$, respectively.

\section{Discussion}

In this study, a selection of parameters that could potentially influence the feeding rate of $A n$. farauti s.s. colony mosquitoes in DMFAs were investigated to identify the 
Table 1 Mosquito feeding rate according the feeding parameters being tested

\begin{tabular}{|c|c|c|c|c|c|c|}
\hline Feeding parameters & $\begin{array}{l}\text { Total number of } \\
\text { mosquitoes in cups }\end{array}$ & $\begin{array}{l}\text { Total number } \\
\text { fed }\end{array}$ & Total unfed & $\begin{array}{l}\text { Empirical average } \\
\text { feeding rate }(\%)^{*}\end{array}$ & Range (\%) & $p$ value $* *$ \\
\hline \multicolumn{7}{|l|}{ Starving time } \\
\hline $\mathrm{Oh}$ & 916 & 240 & 676 & 27 & $4-45$ & $<0.01$ \\
\hline $2 \mathrm{~h}$ & 864 & 288 & 576 & 34 & $15-56$ & $<0.01$ \\
\hline $4 \mathrm{~h}$ & 933 & 368 & 565 & 40 & $20-54$ & $<0.05$ \\
\hline $6 \mathrm{~h}$ & 954 & 405 & 549 & 45 & $23-82$ & $<0.01$ \\
\hline Overnight ( 21 h) & 841 & 492 & 349 & 60 & 19-97 & Ref \\
\hline \multicolumn{7}{|l|}{ Type of starving } \\
\hline Access to water & 217 & 134 & 83 & 62 & $43-89$ & 0.47 \\
\hline Dry starving & 234 & 167 & 67 & 71 & $31-92$ & Ref \\
\hline \multicolumn{7}{|l|}{ Membrane type } \\
\hline Baudruche & 476 & 389 & 87 & 85 & $70-100$ & $<0.05$ \\
\hline Parafilm & 457 & 268 & 189 & 53 & $42-76$ & Ref \\
\hline \multicolumn{7}{|l|}{ Exposure time } \\
\hline $10 \min$ & 426 & 326 & 100 & 77 & $63-98$ & 0.63 \\
\hline $20 \mathrm{~min}$ & 469 & 368 & 101 & 80 & $43-100$ & 0.61 \\
\hline $30 \mathrm{~min}$ & 386 & 314 & 72 & 81 & $72-89$ & Ref \\
\hline \multicolumn{7}{|l|}{ Mosquito age } \\
\hline 3 days & 631 & 447 & 184 & 75 & $39-96$ & 0.38 \\
\hline 5 days & 606 & 473 & 133 & 81 & $50-93$ & Ref \\
\hline 7 days & 613 & 449 & 164 & 75 & $55-90$ & 0.08 \\
\hline \multicolumn{7}{|l|}{ Light/Dark } \\
\hline Light & 317 & 274 & 43 & 85 & $64-96$ & 0.88 \\
\hline Dark & 326 & 276 & 50 & 84 & $63-96$ & Ref \\
\hline \multicolumn{7}{|l|}{ Volume of blood } \\
\hline $125 \mu \mathrm{L}$ & 289 & 190 & 99 & 65 & $50-88$ & $<0.05$ \\
\hline $250 \mu \mathrm{L}$ & 272 & 229 & 43 & 84 & $67-96$ & 0.54 \\
\hline $500 \mu \mathrm{L}$ & 295 & 256 & 39 & 87 & $77-98$ & Ref \\
\hline \multicolumn{7}{|l|}{ Mosquito density } \\
\hline 20 mosquitoes & 194 & 148 & 46 & 76 & $64-79$ & 0.13 \\
\hline 50 mosquitoes & 480 & 410 & 70 & 85 & $79-87$ & Ref \\
\hline 100 mosquitoes & 927 & 696 & 231 & 75 & $67-74$ & $<0.01$ \\
\hline \multicolumn{7}{|c|}{ Water bath temperature } \\
\hline $34^{\circ} \mathrm{C}$ & 330 & 271 & 59 & 82 & 51-95 & 0.23 \\
\hline $38^{\circ} \mathrm{C}$ & 335 & 299 & 36 & 89 & $81-100$ & Ref \\
\hline $42{ }^{\circ} \mathrm{C}$ & 320 & 277 & 43 & 86 & $72-98$ & 0.61 \\
\hline $46^{\circ} \mathrm{C}$ & 305 & 244 & 61 & 79 & 58-96 & 0.09 \\
\hline
\end{tabular}

Ref: reference group for the calculation of the $p$ values

*Empirical averages were calculated as the average feeding rate of all replicates obtained for a specific condition

**Significantly different from the reference when $p<0.05$. Data were transformed using arcsine ( $p$ ) prior to doing the paired $t$ test

optimal conditions to enable high feeding rates. By systematically varying individual parameters sequentially, the baseline feeding rate of $\sim 50 \%$ was increased to $\sim 85 \%$.

Two parameters in particular were associated with improved feeding rates: the starving duration prior to membrane feeding and the membrane type used during the feed. Increasing the starving duration to overnight $(\sim 21 \mathrm{~h})$ resulted in a statistically significant difference in the feeding rate $(60 \%)$ in comparison to the other starving durations (27-45\%). We selected overnight starving, as there were no significant differences between the $6 \mathrm{~h}$ and $4 \mathrm{~h}$ or $2 \mathrm{~h}(p=0.51, p=0.10)$, which had lower feeding rates. However, we did observe a significantly higher mortality rate with overnight starving (7\%) as compared to the other starving times (1\%). This may be due to the long hours of starving. However, the high feeding rate 

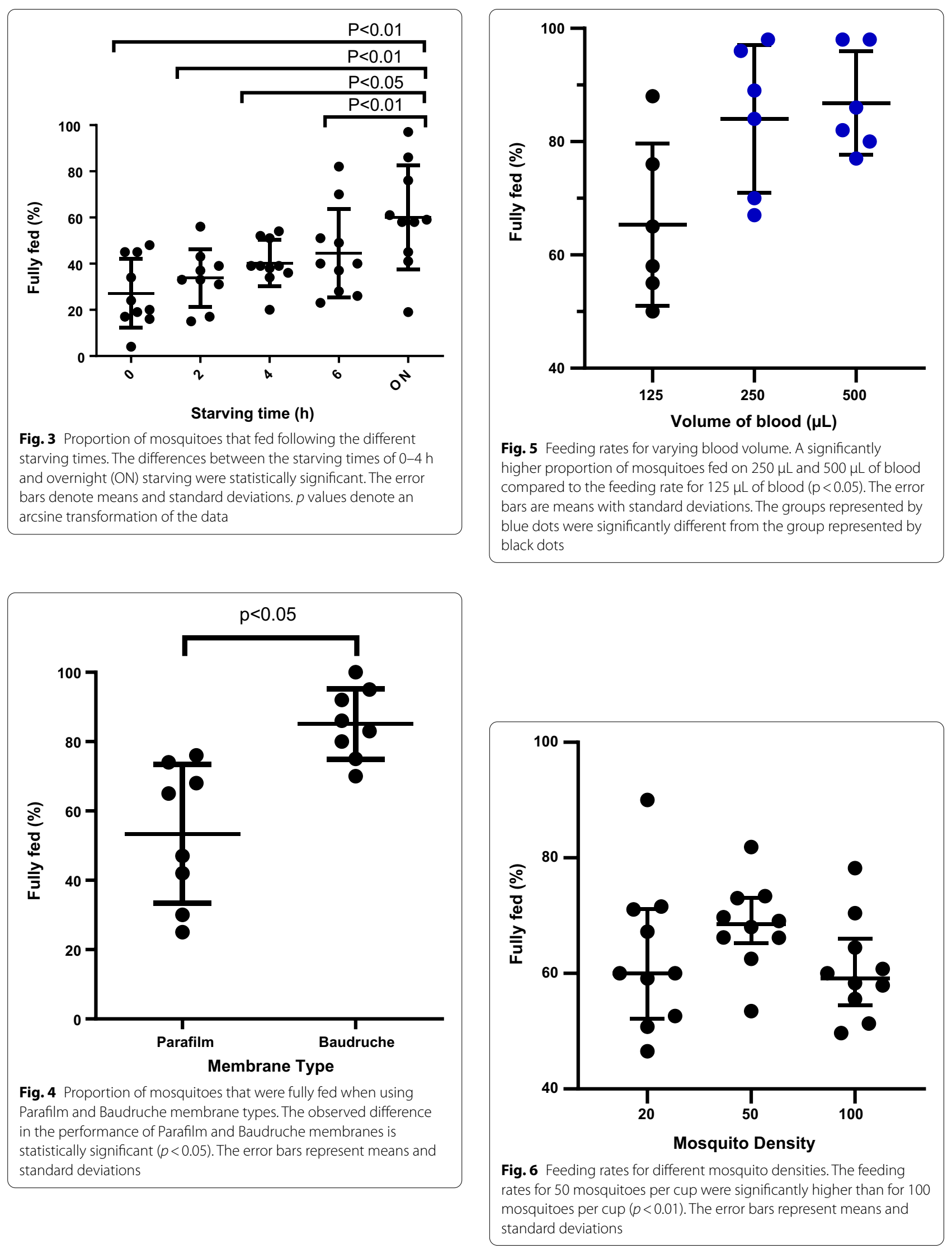
compensates for the increased mortality rate. When compared to the average feeding rate of the other starving times $(2 \mathrm{~h}, 4 \mathrm{~h}$ and $6 \mathrm{~h})$ of $39 \%$, overnight starving resulted in a higher feeding rate by a factor of 1.5. Overnight starving was used in several previous studies [13, $16,22,27,30]$, while some studies used a minimum of $5 \mathrm{~h}$ of starvation with various Anopheles mosquito species $[12,20]$. The use of a Baudruche membrane together with overnight starving further increased the feeding rate to $85 \%$. Previous studies have used either Baudruche membrane or Parafilm for performing DMF [11, 16, 2022 ] with different mosquito species. A study by Coulibaly and colleagues comparing the two membrane types using An. coluzzii mosquitoes showed that there was no significant difference between the Baudruche and Parafilm membranes when adjusting for other covariates [13]. The superior performance of Baudruche membrane over Parafilm observed here may be due in particular to the fact that the Baudruche membrane is made from a natural material, as previous evidence has shown that natural membranes have better feeding performance [31]. Another possibility is that the natural membrane was favoured because it more closely resembled the direct skin feeding, which is used to feed the mosquitoes for colony maintenance. Varying the conditions of the other feeding parameters (e.g., feeding in the light versus in the dark, mosquito age, blood volume, feeding duration and water bath temperature) did not significantly increase the feeding rate further.

Even though there was no significant increase in the feeding rates when testing the other parameters, our chosen selection contributed towards the economical use of resources. It was observed that feeding mosquitoes for $10 \mathrm{~min}$ yielded similar feeding rates as feeding for 20-30 min. This is within the range of feeding times between 10 and $30 \mathrm{~min}$ that have been used in different studies, and represents a significant time saving [11, 21, 30, 32]. We chose to use 10-20 min depending on our feeding schedule. The optimal volume of blood used per glass feeder is important, especially when working with limited amounts of infected blood. A volume of 350 $\mu \mathrm{L}$ was recommended for the size $(2.5 \mathrm{~cm}$ in diameter, surface area of $\sim 5 \mathrm{~cm}^{2}$ ) of the glass feeders used in the present study [11], while another study used a total of $1.5 \mathrm{~mL}$ [20] using the same size feeders. However, volumes between 250 and $500 \mu \mathrm{L}$ yielded similar high feeding rates. As such, we chose to use any volume within the range of $250-500 \mu \mathrm{L}$, depending on the total volume of blood available to feed the mosquitoes. This represents a threefold decrease in the volume of blood used compared to the original volume of $750 \mu \mathrm{L}$.

With respect to mosquito density, it was observed that 50 mosquitoes (approximate density per cup of 1 mosquito $/ 6.8 \mathrm{~cm}^{2}$ ) feeding on a $\sim 5 \mathrm{~cm}^{2}$ membrane surface area yielded a high feeding rate, while 100 mosquitoes per cup (i.e., 1 mosquito $/ 3.4 \mathrm{~cm}^{2}$ ) resulted in lower feeding rates. The difference was statistically significant $(p<0.01)$. There was no significant difference in the feeding rates when comparing densities of 20 mosquitoes and 50 mosquitoes per cup. Interestingly there was also no significant difference between 20 and 100 mosquitoes per cup. This observation could imply that there is really no significant difference between the three mosquito densities, as indicated by the ANOVA test $(F(2,15)=3.861$, $p=0.052$ ). However, the $p$ value observed here is approaching statistical significance, indicating that more tests need to be conducted in order to conclude whether the difference observed between the mosquito densities is significant. Established protocols recommend using 50-100 mosquitoes for similar-sized cups depending on the size of the feeder and the feeding rate $[11,12]$. Based on these results, we chose to use 50 mosquitoes per cup to maximize the number of mosquitoes exposed to blood and reduce the risk of a crowding effect during feeding. Although various groups have preferred using one or the other of the two types of starving conditions, either dry or by exposing the mosquitoes to water prior to feeding $[12,14-18]$, we did not observe any significant difference between the two. It may be that An. farauti s.s. mosquitoes do not feed on water as efficiently as they would on sugar solution, as the mosquitoes have specialized sensory organs that detect the presence of blood or nectar, triggering them to feed [33]. Hence, we observed no significant difference between the feeding rates of the starving conditions. We opted for dry starving.

We also did not observe any significant difference between feeding the mosquitoes when exposed to light or in the dark. However, other studies indicated that their membrane feeding experiments were performed in the dark [20, 22]. Also, a protocol by Ouedraogo and colleagues indicated that DMF should be performed in the dark to mimic the natural feeding conditions during the night, while membrane feeding experiments are commonly undertaken during the day [12]. Our contrasting observation may be because this mosquito species has been colonized for over 50 years and feeds well regardless of the light conditions. Usually in the wild the $A n$. farauti s.s. mosquito would feed in the evening starting at $6 \mathrm{pm}$ and peak between 10 and $11 \mathrm{pm}$ when it is dark [34]. Here we chose to feed the mosquitoes while exposing them to the light, as it is easier to monitor the progress of the mosquitoes feeding.

Furthermore, we did not detect any significant differences in the feeding rates between age groups 3-, 5- and 7-day-old mosquitoes. In contrast, Coulibaly and colleagues noted a significant difference in the feeding rates 
between 3 days and 6-9-day-old An. coluzzii. It could be that this age-dependent behaviour change is species- or colony-specific. Collectively, studies on various Anopheles mosquito species reported using mosquitoes aged $2-8$ days $[6,7,12,13,16,17,23-25]$. Here we chose to work with 3-5-day-old mosquitoes to ensure that we achieved high survival rates on day 7 for the dissection for oocysts and day 14 for the dissection for sporozoites. Finally, we did not observe any significant difference between the water bath temperatures of $34{ }^{\circ} \mathrm{C}, 38{ }^{\circ} \mathrm{C}$, $42{ }^{\circ} \mathrm{C}$ and $46^{\circ} \mathrm{C}$. While most studies have indicated using a water bath at $37^{\circ} \mathrm{C}[18,30]$, this parameter has not been investigated before. Our results show that mosquitoes were able to feed efficiently regardless of fluctuations in the water bath temperatures. We chose to use a water bath temperature of $37-38{ }^{\circ} \mathrm{C}$, as it closely resembles the human body temperature and will be most conducive for malaria parasite survival.

\section{Conclusion}

By sequentially and systematically varying individual membrane feeding parameters, the blood feeding rate of Anopheles farauti s.s. colony mosquitoes was increased significantly to $85 \%$. This highlights the importance of parameter selection and optimization in direct membrane feeding assays. Further work will need to be performed with infected blood to ensure that these parameters result in high infection and survival rates for An. farauti s.s. colony mosquitoes using DMFA.

\section{Abbreviations}

PNG: Papua New Guinea; DMFA: Direct membrane feeding assay; ON: Overnight; An. farauti: Anopheles farauti; An. coluzzii: Anopheles coluzzii.

\section{Supplementary Information}

The online version contains supplementary material available at https://doi. org/10.1186/s13071-021-04842-y.

Additional file 1: Table S1. Flow chart of the feeding parameters that were tested progressively. The selected parameters are in bold and were used in the subsequent tests

\section{Acknowledgements}

We would like to thank the following hardworking staff at the Papua New Guinea Institute of Medical Research, Entomology Laboratory who helped in rearing the An. farauti s.s. colony mosquitoes, particularly Hega Sakel, Siub Yabu and Susie Ibam. We would like to thank Dr. Jetsumon Sattabongkot Prachumsri at Mahidol University, Faculty of Tropical Medicine, for providing the glass membrane feeders.

\section{Authors' contributions}

Designed the study: LT, SK. Conducted the laboratory work: LT. Secured funding: SK. Drafting and preparation of the manuscript: LT, SK. Critically revising the manuscript: SK, RV, MK, TB, LS. All authors read and approved the final manuscript.

\section{Funding}

This study was funded by the National Health and Medical Research Council (NHMRC) of Australia. SK is supported by a Career Development Fellowship from NHMRC of Australia; Lincoln Timinao is supported by a Ph.D. scholarship from James Cook University.

\section{Availability of data and materials}

The raw data pertaining to this manuscript can be obtained from the corresponding author upon request.

\section{Declarations}

\section{Ethics approval and consent to participate}

This study was approved by the Papua New Guinea Medical Research Advisory Council (MRAC 16.26) and the PNGIMR Institutional Review Board (1516).

\section{Consent for publication}

Not applicable.

\section{Competing interests}

The authors declare that they have no competing interests.

\section{Author details}

${ }_{1}^{1}$ Papua New Guinea Institute of Medical Research, Madang, Papua New Guinea. ${ }^{2}$ Australian Institute of Tropical Health and Medicine, James Cook University, Smithfield, QLD 4870, Australia.

Received: 8 March 2021 Accepted: 11 June 2021

Published online: 07 July 2021

\section{References}

1. Smith RC, Vega-Rodríguez J, Jacobs-Lorena M. The Plasmodium bottleneck: malaria parasite losses in the mosquito vector. Mem Inst Oswaldo Cruz. 2014;109(5):644-61.

2. Sauerwein RW, Bousema T. Transmission blocking malaria vaccines: assays and candidates in clinical development. Vaccine. 2015;33(52):7476-82.

3. Rutledge LC, Ward RA, Gould DJ. Studies on the feeding response of mosquitoes to nutritive solution in a new membrane feeder. Mosq News. 1964;24:407-19.

4. Graves PM, Burkot TR, Carter R, Cattani JA, Lagog M, Parker J, Brabin BJ, Gibson FD, Bradley DJ, Alpers MP. Measurement of malarial infectivity of human populations to mosquitoes in the Madang area, Papua New Guinea. Parasitology. 1988;96(Pt 2):251-63.

5. Hisaeda $\mathrm{H}$, Yasutomo K. Development of malaria vaccines that block transmission of parasites by mosquito vectors. J Med Investig. 2002:49(3-4):118-23.

6. Ubalee R, Kim H-C, Schuster AL, McCardle PW, Phasomkusolsil S, Takhampunya R, Davidson SA, Lee W-J, Klein TA. Vector competence of Anopheles kleini and Anopheles sinensis (Diptera: Culicidae) from the Republic of Korea to Vivax malaria-infected blood from patients from Thailand. J Med Entomol. 2016:53(6):1425-32.

7. Kiattibutr K, Roobsoong W, Sriwichai P, Saeseu T, Rachaphaew N, Suansomjit C, Buates S, Obadia T, Mueller I, Cui L, et al. Infectivity of symptomatic and asymptomatic Plasmodium vivax infections to a Southeast Asian vector, Anopheles dirus. Int J Parasitol. 2017:47(2-3):163-70.

8. Olayemi IK, Ande AT, Danlami G. Abdullahi U. Influence of blood meal type on reproductive performance of the malaria vector, Anopheles gambiae s.s. (Diptera:Culicidae). J Entomol. 2011;8(5):459-67.

9. Taylor PJ, Hurd H. The influence of host haematocrit on the blood feeding success of Anopheles stephensi: implications for enhanced malaria transmission. Parasitology. 2001;122(Pt 5):491-6.

10. Galun R, Koontz LC, Gwadz RW. Engorgement response of anopheline mosquitoes to blood fractions and artificial solutions. Physiolo Entomol. 1985;10:145-9.

11. Sattabongkot J, Kumpitak C, Kiattibutr K. Membrane feeding assay to determine the infectiousness of Plasmodium vivax gametocytes. Methods Mol Biol. 2015;1325:93-9. 
12. Ouédraogo AL, Guelbéogo WM, Cohuet A, Morlais I, King JG, Gonçalves BP, Bastiaens GJH, Vaanhold M, Sattabongkot J, Wu Y, et al. A protocol for membrane feeding assays to determine the infectiousness of $P$. falciparum naturally infected individuals to Anopheles gambiae. Malaria World J. 2013:4(16):1-4

13. Coulibaly MB, Gabriel EE, Sinaba Y, Sylla D, Sacko A, Sylla L, Coulibaly B, Hume JCC, Baber I, Assadou MH, et al. Optimizing direct membrane and direct skin feeding assays for Plasmodium falciparum transmissionblocking vaccine trials in Bancoumana, Mali. Am J Trop Med Hyg. 2017;97(3):719-25.

14. Abduselam N, Zeynudin A, Berens-Riha N, Seyoum D, Pritsch M, Tibebu H, Eba K, Hoelscher M, Wieser A, Yewhalaw D. Similar trends of susceptibility in Anopheles arabiensis and Anopheles pharoensis to Plasmodium vivax infection in Ethiopia. Parasit Vectors. 2016;9(1):552.

15. Da DF, Churcher TS, Yerbanga RS, Yameogo B, Sangare I, Ouedraogo JB, Sinden RE, Blagborough AM, Cohuet A. Experimental study of the relationship between Plasmodium gametocyte density and infection success in mosquitoes; implications for the evaluation of malaria transmissionreducing interventions. Exp Parasitol. 2015;149:74-83.

16. Rios-Velásquez CM, Martins-Campos KM, Simões RC, Izzo T, dos Santos EV, Pessoa FA, Lima JB, Monteiro WM, Secundino NF, Lacerda MV, et al. Experimental Plasmodium vivax infection of key Anopheles species from the Brazilian Amazon. Malar J. 2013;12:460.

17. Zhu G, Xia H, Zhou H, Li J, Lu F, Liu Y, Cao J, Gao Q, Sattabongkot J. Susceptibility of Anopheles sinensis to Plasmodium vivax in malarial outbreak areas of central China. Parasit Vectors. 2013;6:176.

18. Sattabongkot J, Maneechai N, Phunkitchar V, Eikarat N, Khuntirat B, Sirichaisinthop J, Burge R, Coleman RE. Comparison of artificial membrane feeding with direct skin feeding to estimate the infectiousness of Plasmodium vivax gametocyte carriers to mosquitoes. Am J Trop Med Hyg. 2003;69(5):529-35.

19. Novak MG, Berry WJ, Rowley WA. Comparison of four membranes for artificially bloodfeeding mosquitoes. J Am Mosq Control Assoc. 1991;7(2):327-9.

20. Awono-Ambene HP, Diawara L, Robert V. Comparison of direct and membrane feeding methods to infect Anopheles arabiensis with Plasmodium falciparum. Am J Trop Med Hyg. 2001;64(1-2):32-4.

21. Kiattibutr K, Roobsoong W, Sriwichai P, Saeseu T, Rachaphaew N, Suansomjit C, Buates S, Obadia T, Mueller I, Cui L, et al. Infectivity of symptomatic and asymptomatic Plasmodium vivax infections to a Southeast Asian vector, Anopheles dirus. Int J Parasitol. 2017;47(2-3):163-70.

22. Bonnet S, Gouagna C, Safeukui I, Meunier JY, Boudin C. Comparison of artificial membrane feeding with direct skin feeding to estimate infectiousness of Plasmodium falciparum gametocyte carriers to mosquitoes. Trans R Soc Trop Med Hyg. 2000;94(1):103-6.
23. Vallejo AF, Rubiano K, Amado A, Krystosik AR, Herrera S, Arévalo-Herrera M. Optimization of a membrane feeding assay for Plasmodium vivax infection in Anopheles albimanus. PLoS Negl Trop Dis. 2016;10(6):e0004807.

24. Witmer K, Sherrard-Smith E, Straschil U, Tunnicliff M, Baum J, Delves M. An inexpensive open source 3D-printed membrane feeder for human malaria transmission studies. Malar J. 2018;17(1):282.

25. Bousema T, Dinglasan RR, Morlais I, Gouagna LC, van Warmerdam T, Awono-Ambene PH, Bonnet S, Diallo M, Coulibaly M, Tchuinkam T, et al. Mosquito feeding assays to determine the infectiousness of naturally infected Plasmodium falciparum gametocyte carriers. PLoS One. 2012;7(8):e42821.

26. Stone WJ, Eldering M, van Gemert GJ, Lanke KH, Grignard L, van de VegteBolmer MG, Siebelink-Stoter R, Graumans W, Roeffen WF, Drakeley CJ, et al. The relevance and applicability of oocyst prevalence as a read-out for mosquito feeding assays. Sci Rep. 2013;3:3418.

27. Vallejo AF, Rubiano K, Amado A, Krystosik AR, Herrera S, Arevalo-Herrera M. Optimization of a membrane feeding assay for Plasmodium vivax infection in Anopheles albimanus. PLoS Negl Trop Dis. 2016;10(6):e0004807.

28. Sweeney AW. Larval salinity tolerances of the sibling species of Anopheles farauti. J Am Mosq Control Assoc. 1987;3(4):589-92.

29. Zar JH. Biostatistical Analysis. 5th ed. Essex: Pearson Education; 2014.

30. Diallo M, Toure AM, Traore SF, Niare O, Kassambara L, Konare A, Coulibaly M, Bagayogo M, Beier JC, Sakai RK, et al. Evaluation and optimization of membrane feeding compared to direct feeding as an assay for infectivity. Malar J. 2008;7:248

31. Novak MG, Berry WJ, Rowley WA. Comparison of four membranes for artificially bloodfeeding mosquitoes. J Am Mosq Control Assoc. 1991;7:327-9.

32. Damiens D, Soliban SM, Balestrino F, Alsir R, Vreysen MJ, Gilles JR. Different blood and sugar feeding regimes affect the productivity of Anopheles arabiensis colonies (Diptera: Culicidae). J Med Entomol. 2013;50(2):336-43.

33. Jové V, Gong Z, Hol FJH, Zhao Z, Sorrells TR, Carroll TS, Prakash M, McBride CS, Vosshall LB. Sensory discrimination of blood and floral nectar by Aedes aegypti mosquitoes. Neuron. 2020;108(6):1163-1180.

34. Reimer LJ, Thomsen EK, Koimbu G, Keven JB, Mueller I, Siba PM, Kazura JW, Hetzel MW, Zimmerman PA. Malaria transmission dynamics surrounding the first nationwide long-lasting insecticidal net distribution in Papua New Guinea. Malar J. 2016;15:25.

\section{Publisher's Note}

Springer Nature remains neutral with regard to jurisdictional claims in published maps and institutional affiliations.
Ready to submit your research? Choose BMC and benefit from:

- fast, convenient online submission

- thorough peer review by experienced researchers in your field

- rapid publication on acceptance

- support for research data, including large and complex data types

- gold Open Access which fosters wider collaboration and increased citations

- maximum visibility for your research: over $100 \mathrm{M}$ website views per year

At BMC, research is always in progress.

Learn more biomedcentral.com/submissions 